5th International Workshop on Astronomy and

Relativistic Astrophysics (IWARA2011)

International Journal of Modern Physics: Conference Series

Vol. 18 (2012) 136-139

(C) World Scientific Publishing Company

DOI: $10.1142 / \mathrm{S} 2010194512008343$

\title{
DATA ANALYSIS OF GRAVITATIONAL WAVES SIGNALS FROM MILLISECONDS PULSARS
}

\author{
F. G. OLIVEIRA, R. M. MARINHO, Jr. and J. G. COELHO \\ Departamento de Física, Instituto Tecnológico de Aeronáutica, \\ Praça Marechal Eduardo Gomes, 50 - Vila das Acácias, \\ São José dos Campos, 12228-900, Brasil \\ fe.fisica@gmail.com \\ N. S. MAGALHAES \\ Universidade Federal de São Paulo, Campus Diadema, \\ R. Prof. Artur Riedel 275, Diadema, SP 09972-270, Brasil
}

\begin{abstract}
The present work is devoted to the detection of monochromatic gravitational waves signals from pulsars using the ALLEGRO's data detector. In this work we will present the region (in frequency) of millisecond pulsars of the 47 Tucanae (NGC 104) in the band of detector. According with this result was possible to analyse the frequency of the pulsar J1342+2822c, searching annual Doppler variations using power spectrum estimates for the year 1999. We tested this method injecting a simulated signal in real data and we were able to detect it.
\end{abstract}

Keywords: Data analysis; Gravitational Waves; Pulsar

\section{Introduction}

We present the region of 47 Tucanae pulsars that are in the band of the ALLEGRO's detector and we compared with the ALLEGRO noise sensitivity curve (in units of strain $/ \sqrt{\mathrm{Hz}}$ ). The focus of this work was to analyse ALLEGRO's data ${ }^{1}$ for the year 1999 taking into account the effect due to the orbital motion of the Earth for specific frequencies, $923.36 \mathrm{~Hz}$ that correspond of the pulsars 47 Tucanae (NGC 104) named as J1342+2822c. This analysis was based on estimates of power spectrum of the data using averaged modified periodograms ${ }^{2}$ which reinforce the presence of peaks due to monochromatic signals.

\section{The Characteristic Amplitude of Pulsar's GW}

Pulsars with non-axisymmetric rotation emit monochromatic gravitational wave signals (MGW). The amplitude of gravitational waves emitted by a rotating neutron stars (NS) can be expressed in terms of the angular frequancy $w_{G}$ of the gravitational wave generated by the pulsar, that is twice its angular frequancy, the distance to the 
Earth $r$, the moment of inertia $I$ and the ellipticity $\epsilon$ resulting from the distortion process $\mathrm{as}^{3}$ :

$$
h_{c}=7.721 \times 10^{-25}\left(\frac{\mathrm{kpc}}{r}\right)\left(\frac{\omega_{G}}{2 \pi 1000 \mathrm{~Hz}}\right)^{2}\left(\frac{I}{10^{38} \mathrm{kgm}^{2}}\right)\left(\frac{\epsilon}{10^{-6}}\right) .
$$

Its value depends on the physical mechanism that makes the star non-axisymmetric and is highly uncertain.

\section{The Strain-Noise Sensitivity of ALLEGRO}

We present the region of MGW signals from pulsars in strain-noise spectrum of ALLEGRO in the Fig. (1). This figure shows the gravitational strain $h_{c}$ for the known pulsars in the band of ALLEGRO from the ATNF catalog. This quantity was derived assuming that all observed spin-down is due to energy loss caused by emission of gravitational radiation (and no other braking mechanisms ${ }^{4,5}$ ). The strain for the pulsars is compared with the noise sensitivity curve for the ALLEGRO's detector (both in units of strain $/ \sqrt{\mathrm{Hz}}$ ).

\section{Determination of the observation time $\Delta t$}

For the purposes of the present analysis we are interested only in annual Doppler shift, so we need to choose an observation time $\Delta t$ such that the diurnal Doppler shift $\Delta \nu_{d}$ remains in the same frequency bin $\Delta f=1 / \Delta t$. The minimum $\Delta f_{\text {min }}$ corresponds to the maximum diurnal Doppler shift, $\Delta \nu_{d \max }=\Delta f_{\min }=1 / \Delta t_{\max }$.

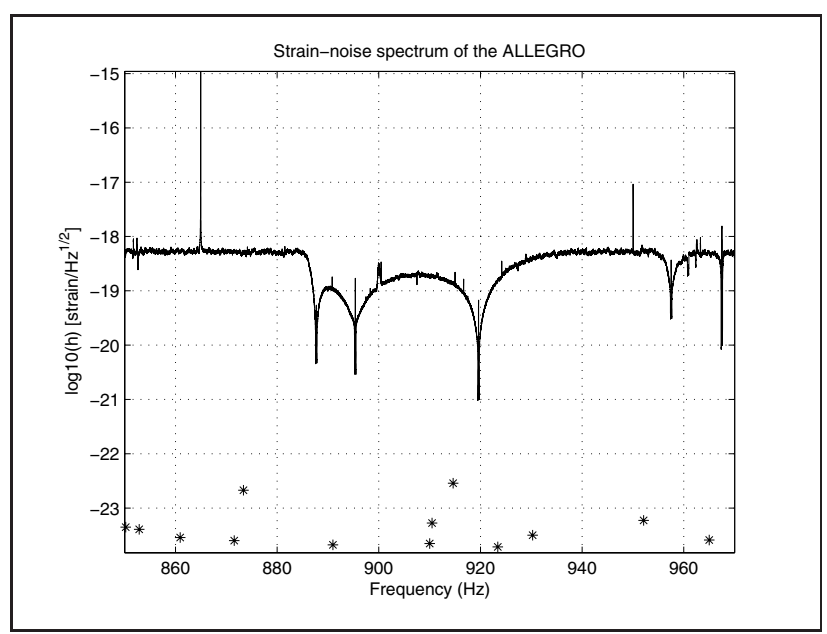

Fig. 1. The figure shows the gravitational strain $h_{c}$ for the known pulsars for thirteen millisecond pulsars in 47 Tucanae (NGC 104). The strain $h_{c}$ is compared with the strain-noise spectrum of the detector ALLEGRO (both in units of strain $/ \sqrt{\mathrm{Hz}}$ ). 
The maximum diurnal Doppler shift arrives when the Earth and the star are in the line of nodes 6 .

$$
\Delta \nu_{d \max }=\nu_{s} \frac{2 w r}{c}
$$

where $w$ and $r$ are the angular rotation the radius of the Earth respectively and $\nu_{s}$ is the pulsar frequency $923.36 \mathrm{~Hz}(\mathrm{~J} 1342+2822 \mathrm{c})$. The annual Doppler shift in a full year of observation is,

$$
\Delta \nu_{a}=\nu_{s} \frac{2 R \Omega}{c}
$$

In order to eliminate the daily Doppler shift, we used $\Delta t=350 \mathrm{~s}$ for the observation time in our data analysis.

\section{The Data Analysis}

The goal of the analysis was to search for an annual Doppler shift in the ALLEGRO's data. For this we stablished a observation time $\Delta t=350 \mathrm{~s}$ so that the frequency of a possible observed signal will not change from one bin to another during the day. We have taken the power spectral density for 100 days of the year $1999^{7}$. We fixed our attention in the bins that contains the frequency of $923.36 \mathrm{~Hz}$ that corresponds to the GW irradiated by the pulsar J1342+2822c, looking for an excess energy in these bins during these 100 days. We have chosen this two pulsar because their irradiated frequency is near the most sensible frequency of the detector ${ }^{1}$. We simulated a GW signal with adimensional amplitude of the $h=2.6 \times 10^{-17}$ and added it to ALLEGRO's data. The result for this analysis is in the Fig. (2).

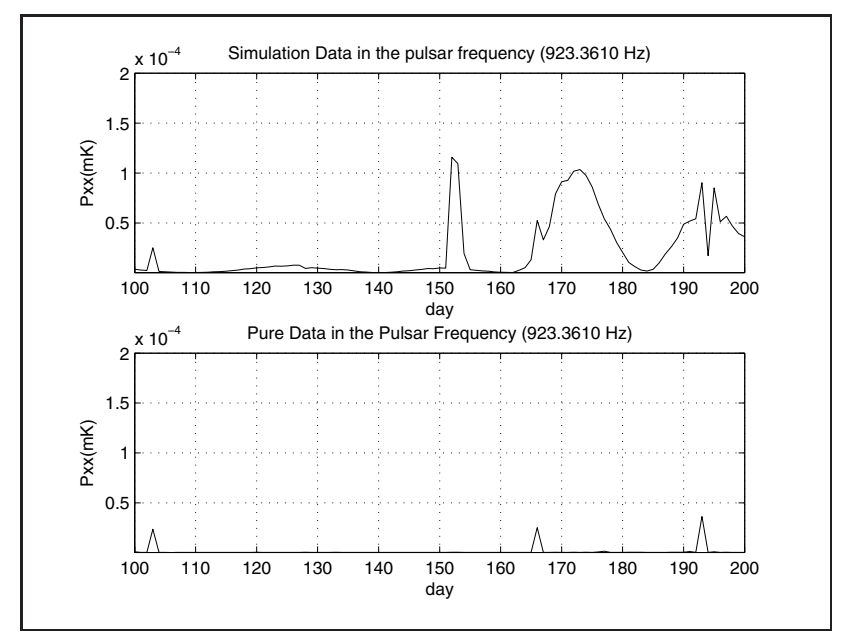

Fig. 2. Upper Figure: Variation of the power spectrum for the simulated signal added to the data. Lower Figure: Variation of the power spectrum for raw data in the frequency of the pulsar $\mathrm{J} 1342+2822 \mathrm{c}$. 


\section{Conclusions}

In this analysis we can not identify any behavior of the modulations Doppler signals for the real data. But was possible to test our data analysis for the monochromatic gravitational waves signals. We detected the simulated signal buried in the noise. The detection probability using the Neyman-Pearson criterion will be published in the next paper.

\section{References}

1. E. Mauceli., P. M. McHugh., W. O. Hamilton, W. W. Johnson and A. Morse, it Phys. Rev. D 54, (1996) 1264-1275.

2. F. G. Oliveira et al., Search for monochromatic signals using data from the ALLEGRO gravitational wave detector. Inter. Journ. of Mod. Phys. Dv. 19 p. 1293-12972010

3. S. Hawding and W. Israel; 300 Years of Gravitation; Cambridge University Press (1987).

4. Santostasi, G. Upper and lower limits on the Crab pulsars astrophysical parameters set from gravitational wave observations by LIGO: braking index and energy considerations.. ArXiv:gr-qc/0807.2485v1, July 16, 2008.

5. Gourgoulhon, E. and Bonazzola, S. Gravitational Waves from isolated neutron stars. ArXiv:astro-ph/9605150v1, May 24, 1996.

6. F. G. Oliveira et al., Data Analysis of Monochromatic Signals from ALLEGRO GW Detector. Nuclear Physics. B, Proceedings Supplement (Print)v. 199 p. 353-3562010

7. P. D. Welch., it IEEE Trans. and Audio Electroacoust., AU-15, 70-73, June 1970. 